\title{
Flora y vegetación de la marisma de Doñana en el marco del proyecto de restauración ecológica Doñana 2005
}

\author{
P. García-Murillo ${ }^{1, *}$, R. Fernández-Zamudio ${ }^{1}$, S. Cirujano ${ }^{2}$, A. Sousa ${ }^{1}$, I. Nieto ${ }^{1}$, J. Andújar ${ }^{1}$ \\ y D. León ${ }^{1}$ \\ ${ }^{1}$ Dept. Biología Vegetal y Ecología. Facultad de Farmacia. Univ. Sevilla. C/Prof. García González s/n. 41012 \\ Sevilla. España. \\ ${ }^{2}$ Real Jardin Botánico, CSIC. Plaza de Murillo 2. 28014 Madrid. España \\ * Corresponding author: pgarcia@us.es
}

\begin{abstract}
Flora and vegetation of the Doñana marshland in the context of the Doñana 2005 ecological restoration project

Doñana 2005 is an ambitious ecological restoration project that is developed in the wetlands of the Doñana protected space (SW Spain). The objective of this article is to present the preliminary results of the Flora and Vegetation Team linked to the project. Floristic spectrum and phytogeographical elements from the flora of the study area are shown. Furthermore, data corresponding to the evolution of the land cover vegetation of the studied zones is offered.
\end{abstract}

Key words: Ecological restoration, Doñana, flora, vegetation, marshes, SW Europe.

\section{RESUMEN}

Flora y vegetación de la marisma de Doñana en el marco del proyecto de restauración ecológica Doñana 2005

Doñana 2005 es un ambicioso proyecto de restauración ecológica que se desarrolla en el los humedales del espacio protegido de Doñana (SW España). El objetivo de este artículo es dar a conocer los resultados preliminares del Equipo de Flora y Vegetación vinculado a dicho proyecto. Se muestran el espectro florístico y los elementos fitogeográficos de la flora del área de estudio. Asimismo se ofrecen datos correspondientes a la evolución de la cubierta vegetal de las zonas estudiadas.

Palabras clave: Restauración ecológica, Doñana, flora, vegetación, marismas, SW Europa.

\section{INTRODUCCIÓN}

Doñana 2005 es un proyecto del Ministerio de Medio Ambiente que pretende la regeneración ecológica de una parte de las Marismas del Guadalquivir del espacio protegido de Doñana, transformadas a causa de la actividad humana (Narbona, 2006). Se trata de un ambicioso proyecto que abarca importantes obras de restauración ambiental, así como numerosos trabajos de investigación científica vinculados al seguimiento de los efectos de los trabajos de inge- niería en el medio natural. Para más información véase Bayán \& Alonso (2006).

En este artículo se ofrece información sobre los resultados obtenidos hasta la fecha de uno de los equipos de investigación que participan en dicho proyecto: el equipo de Flora y Vegetación acuática, cuyos objetivos particulares son el conocimiento de la flora y vegetación de las zonas de proyecto asignadas, así como de los posibles efectos que sobre estos organismos pudieran tener las tareas de restauración ejecutadas. Se pretende por tanto: proporcionar un catálogo ex- 


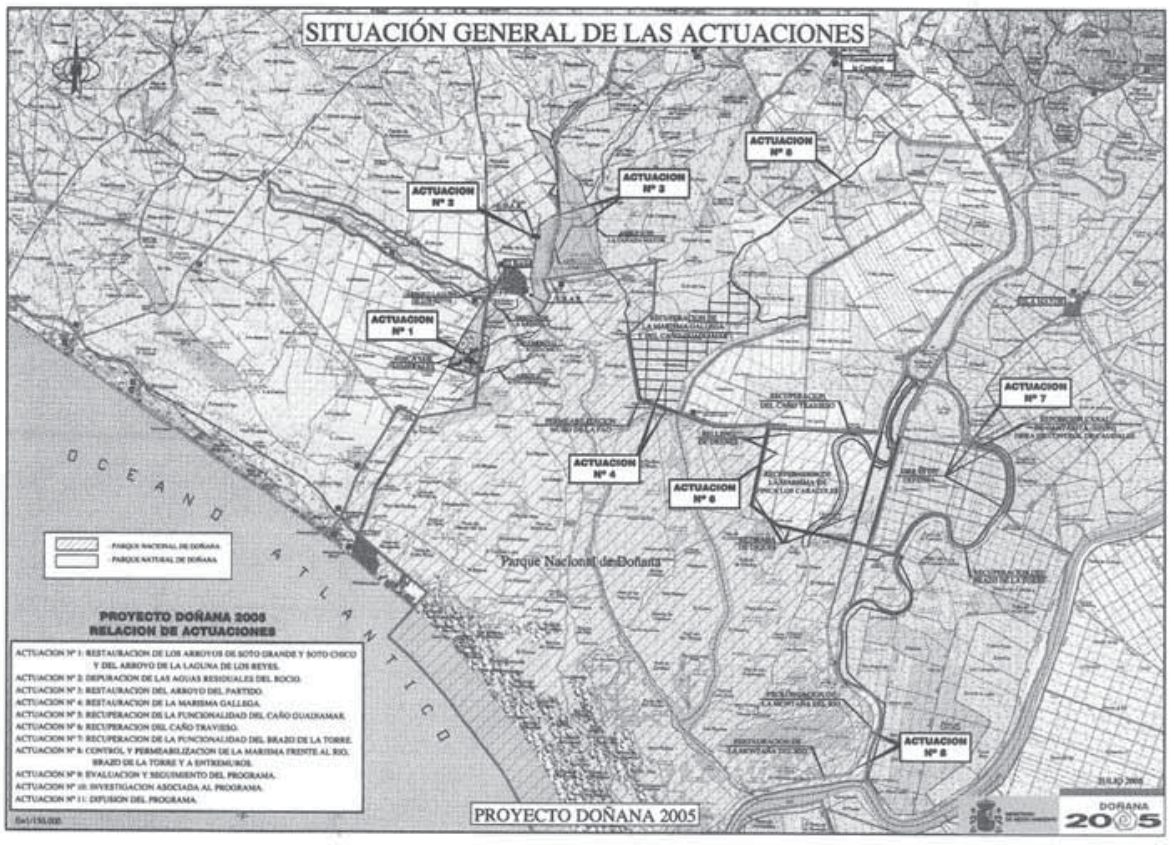

Figura 1. Situación de las actuaciones del Proyecto de Restauración Ecológica Doñana 2005. Location of the actions in Doñana 2005 Ecological Restoration Project.

haustivo de la flora del área de estudio, que sirva de hito, para poder detectar la pérdida o aparición de nuevos táxones vegetales en la zona, y conocer la evolución de la superficie cubierta por los distintos tipos de comunidades vegetales. A partir de ambos objetivos, pensamos, se podrá conocer de forma más precisa la eficacia en las tareas de restauración realizadas.

El área de estudio correspondiente a los trabajos que aquí se exponen incluye las zonas de actuación 4 y 8 del proyecto Doñana 2005 (Fig. 1), ambas incluidas dentro de los ecosistemas de las marismas del Guadalquivir del espacio protegido de Doñana.

Para mayor información sobre las marismas de Doñana ver García Novo (1997) y Marín \& García Novo (2005).

La zona de actuación $n^{0} 4$ (Fig. 1) se sitúa al norte del Parque Nacional de Doñana, en la denominada "Marisma Gallega". Una zona donde, durante los años 70, el Ministerio de Agricultura realizó profundas alteraciones con la finalidad de mejorar la calidad de sus suelos para su puesta en cultivo. A grandes rasgos, el método utilizado para conseguir este fin fue la creación de un complicado sistema de drenaje con el que se pretendía desalinizar los suelos marismeños y, aunque los terrenos nunca se pusieron en cultivo, estos trabajos tuvieron como consecuencia la transformación del relieve y la red de avenamiento de la marisma original. Los trabajos de restauración en esta zona (Bayán \& Alonso, 2006) consisten en la restitución del relieve existente en la época anterior a las transformaciones realizadas por el Ministerio de Agricultura. Para ello se ha actuado en 1800 ha, básicamente en tres direcciones: eliminando los canales artificiales, restableciendo la continuidad entre dos zonas de la Marisma de Doñana separadas (la marisma del Parque Nacional y la Marisma Gallega) y restaurado el perfil original del Caño Guadiamar, que atraviesa la zona.

La zona de actuación $n^{0} 8$ (Fig. 1) se ubica en el este de la marisma del Parque Nacional, en los lugares en que esta limita con el río Guadalquivir y el Brazo de la Torre. Dicha zona (Marisma Salada) presenta un muro de unos 29 km de longitud que impide la comunicación 
entre estos cursos de agua y la marisma. Las obras de restauración en este caso (Bayán \& Alonso, 2006) pretenden la permeabilización de este muro, que en épocas pasadas permitía el encuentro entre estos dos medios acuáticos.

\section{MATERIAL Y MÉTODOS}

Los trabajos encaminados al conocimiento y distribución de la flora de la marisma se realizaron a partir de prospecciones en el campo durante los años 2004-2005. Los ejemplares recolectados se identificaron en el laboratorio siguiendo, básicamente, en el caso de las plantas vasculares, la Flora Vascular de Andalucía Occidental (Valdés, Talavera \& Fernández-Galiano, 1987), en el caso de los briófitos los criterios de Cirujano et al. (1988) y en el de los carófitos a Comelles (1985).

Asimismo, con objeto de considerar el material recolectado por otros botánicos en el área de estudio, se consultaron los herbarios de la Universidad de Sevilla (SEV) y Real Jardín Botánico de Madrid (MA), donde se encuentran depositados la mayor parte del material de herbario correspondiente a Doñana.
Los pliegos de herbario recolectados para este trabajo se han depositado en el herbario de Universidad de Sevilla (SEV).

Respecto a la vegetación, con la intención de poder comparar la evolución de la cubierta vegetal de las zonas de estudio, se sectorizó esta en una serie de unidades de vegetación. Las unidades de vegetación se elaboraron a partir de la caracterización de las comunidades vegetales que se encontraban en la zona de estudio, las cuales se identificaron siguiendo el método sigmatista de Braun Blanquet (1979). La razón principal para elegir este método fue la existencia de un excelente trabajo (Rivas Martínez et al. 1980) que describía la vegetación del Parque Nacional de Doñana siguiendo este sistema y que sirvió de armazón a nuestras investigaciones.

Por otra parte, teniendo en cuenta los resultados obtenidos en investigaciones anteriores (Bernués, 1990; Duarte et al., 1990; Espinar, 2000; Espinar et al., 2002) y con objeto de comprobar los principales factores que organizaban la vegetación en las zonas de estudio se realizaron diversos transectos y se midieron diversas variables fisicoquímicas. Las comunidades reconocidas y ubicadas ecológicamente se agruparon en

Tabla 1. Información técnica sobre las ortofotos utilizadas en la elaboración de la cartografía de la vegetación. Technical information about the ortophotographs used in the elaboration of the vegetation cartography.

\begin{tabular}{|c|c|c|}
\hline Soporte cartográfico & \multicolumn{2}{|c|}{$\begin{array}{l}\text { - M.T.A. E. 10.000 formato digital (Instituto de Cartografía de Andalucía) } \\
\text { - M.T.N. E. 1:50.000 formato digital (Instituto Geográfico Nacional) }\end{array}$} \\
\hline Software digitalización & \multicolumn{2}{|l|}{ ARC-GIS 9.0} \\
\hline Fechas del vuelos & 1998-1999 (julio) & $2001-2002$ \\
\hline $\begin{array}{l}\text { Resolución geométrica de las } \\
\text { ortofotos }\end{array}$ & $1 \mathrm{~m}$ (escala aproximada 1:60.000) & $0,5 \mathrm{~m}$ (escala aproximada 1:20.000) \\
\hline Tipo de película & Color & Pancromática en Blanco/Negro \\
\hline Fuente & $\begin{array}{l}\text { Junta de Andalucía (Consejería de Obras } \\
\text { Públicas y Transporte, Agricultura y } \\
\text { Pesca y Medio Ambiente) }\end{array}$ & $\begin{array}{l}\text { Junta de Andalucía (Consejería de Obras } \\
\text { Públicas y Transporte, Agricultura y } \\
\text { Pesca y Medio Ambiente) }\end{array}$ \\
\hline Hojas M.T.A. fotointerpretadas & $\begin{array}{l}1033-2-2,1033-2-3,1033-2-4,1033-3-1 \\
1033-3-2,1033-3-3,1033-3-4,1033-4-2\end{array}$ & $\begin{array}{l}\text { 1018-2-1, 1018-2-2, 1018-2-3, 1018-3-1, } \\
1018-3-2,1001-3-4\end{array}$ \\
\hline Proyección & \multicolumn{2}{|l|}{ Proyección Transversal de Mercator (UTM) } \\
\hline Elipsoide de referencia & \multicolumn{2}{|c|}{ Elipsoide Internacional de Hayford (Europeam Datum 1950-Ed50) } \\
\hline Origen de coordenadas & \multicolumn{2}{|l|}{ Huso 30} \\
\hline $\begin{array}{l}\text { Escala aproximada de } \\
\text { fotointerpretación }\end{array}$ & \multicolumn{2}{|c|}{$1: 2.500$ (entre $1: 10.000$ y $1: 1.000$ según la zona) } \\
\hline
\end{tabular}




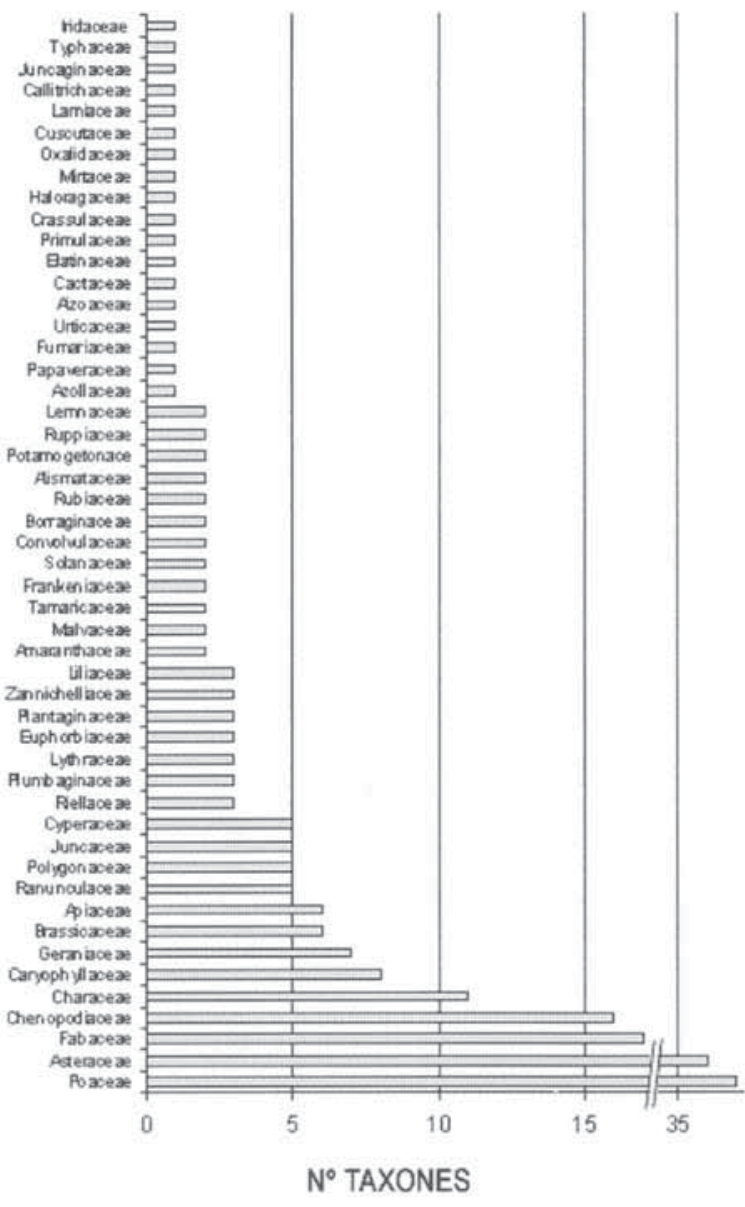

Figura 2. Significación de las distintas familias encontradas en el área de estudio. Significance of the different families found in the study area. unidades de vegetación reconocibles en las ortofotos de la zona.

Finalmente, para poder comparar las situaciones antes y durante la ejecución de las obras se utilizaron, ortofotos correspondientes a los años 1998-1999 (antes de las obras) y 2001-2002 (durante la realización de las obras). Las características técnicas de los fotografías aéreas utilizadas se recogen en la Tabla 1.

\section{RESULTADOS}

La flora catalogada hasta el momento en nuestra zona de estudio está compuesta por un total de 226 taxones, los cuales se reparten de la siguiente forma: la práctica totalidad de ellos pertenecen a la División Spermatophyta (94\%), el resto pertenecen a la División Chlorophyta, representada por especies de la familia Characeae (5\%); la División Bryophyta, que incluye sólo los taxones del género Riella, supone un $1 \%$ y, la División Pteridophyta con un sólo taxón representa menos del $1 \%$.

Por otra parte, dentro de la División Spermatophyta, el grupo vegetal más importante en especies en la zona de estudio, se han encontrado 48 familias. Entre estas: Poaceae, Asteraceae, Fabaceae y Chenopodiaceae son las que aportan mayor número de táxones al catálogo florístico. La figura 2 muestra la distribución de las distintas

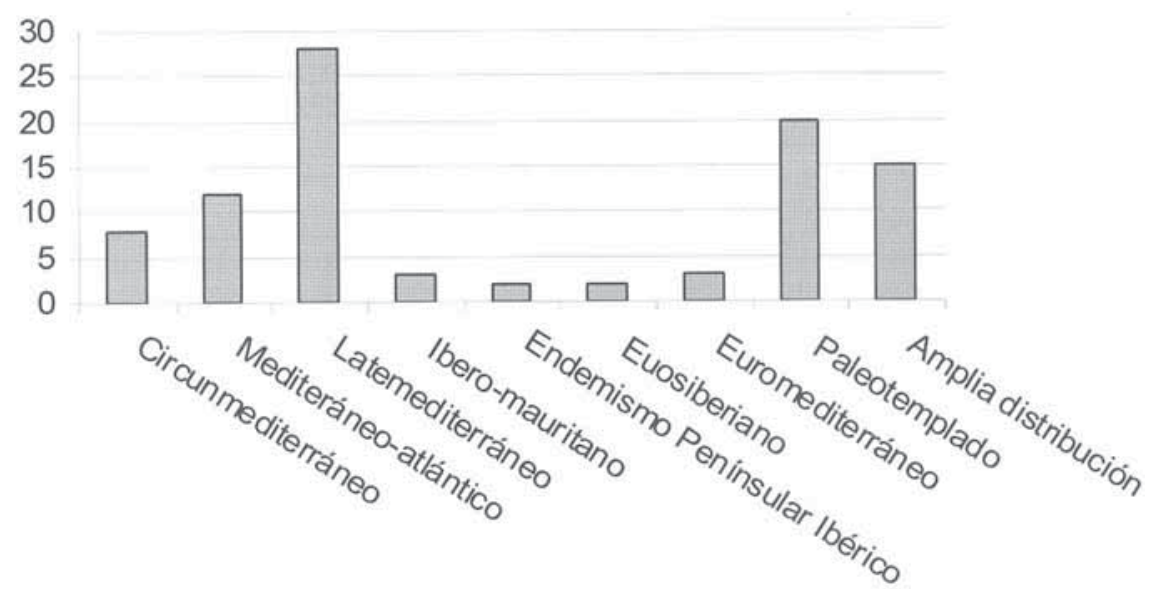

Figura 3. Elementos fitogeográficos en el área de estudio. Phytogeographical elements in the study area. 
Flora y vegetación de Doñana 2005

Tabla 2. Especies vegetales singulares encontradas en el área de estudio. Se incluye la referencia a los autores de los que procede la calificación. Particular vegetal species found in the study area. Reference to the authors who have identified these species is included.

\begin{tabular}{|c|c|c|c|c|c|c|}
\hline $\begin{array}{l}\text { TAXONES } \\
\text { SINGULARES }\end{array}$ & $\begin{array}{l}\text { Cirujano } \\
\text { et al., } 1992\end{array}$ & $\begin{array}{l}\text { Hernández Bermejo } \\
\text { et al., } 1994\end{array}$ & $\begin{array}{l}\text { Blanca } \\
\text { et al., } 2000\end{array}$ & $\begin{array}{l}\text { Cobo } \\
\text { et al., } 2002\end{array}$ & $\begin{array}{l}\text { Bañarés } \\
\text { et al., } 2003\end{array}$ & $\begin{array}{l}\text { Cabezudo } \\
\text { et al. } 2005\end{array}$ \\
\hline $\begin{array}{l}\text { Chara oedophilla } \\
\text { Feldman. }\end{array}$ & $\bullet$ & - & - & - & - & - \\
\hline $\begin{array}{l}\text { Lamprothamnium } \\
\text { papulosum (Wallr.) J. } \\
\text { Groves }\end{array}$ & $\bullet$ & - & - & - & - & - \\
\hline $\begin{array}{l}\text { Nitella hyalina (DC.) } \\
\text { Agardh }\end{array}$ & $\bullet$ & - & - & - & - & - \\
\hline $\begin{array}{l}\text { Tolypella hispanica } \\
\text { Nordst. ex T.F. Allen }\end{array}$ & $\bullet$ & - & - & - & - & - \\
\hline $\begin{array}{l}\text { Tolypella salina } \\
\text { Corillion }\end{array}$ & $\bullet$ & - & - & - & - & \\
\hline $\begin{array}{l}\text { Riella cossoniana } \\
\text { Trabut. }\end{array}$ & $\bullet$ & - & - & - & - & \\
\hline $\begin{array}{l}\text { Riella helicophylla } \\
\text { (Bory \& Mont) Mont. }\end{array}$ & $\bullet$ & - & - & - & - & \\
\hline $\begin{array}{l}\text { Riella notarisii (Mont) } \\
\text { Mont. }\end{array}$ & $\bullet$ & - & - & - & - & - \\
\hline $\begin{array}{l}\text { Ranunculus peltatus } \\
\text { Schrank subsp. fucoides } \\
\text { (Freyn) Muñoz } \\
\text { Garmendia }\end{array}$ & - & $\bullet$ & - & - & - & - \\
\hline $\begin{array}{l}\text { Halopeplis } \\
\text { amplaxicaulis (Valh) } \\
\text { Ung. Stemb. ex Cesati, } \\
\text { Passer \& Gilbelli. }\end{array}$ & - & - & - & $\bullet$ & - & $\bullet$ \\
\hline $\begin{array}{l}\text { Limonium algarvense } \\
\text { Erben }\end{array}$ & - & - & - & $\bullet$ & - & $\bullet$ \\
\hline Lythrum baeticum $\mathrm{L}$ & $\bullet$ & $\bullet$ & - & - & $\bullet$ & $\bullet$ \\
\hline $\begin{array}{l}\text { Callitriche truncata } \\
\text { subsp. occidentalis } \\
\text { (Rouy) Schotsman }\end{array}$ & $\bullet$ & - & - & - & - & - \\
\hline $\begin{array}{l}\text { Carduus bourgeanus } \\
\text { subsp. bourgeanus } \\
\text { Boiss. \& Reuter }\end{array}$ & - & $\bullet$ & - & $\bullet$ & - & - \\
\hline $\begin{array}{l}\text { Leontodon maroccanus } \\
\text { (Pers.) Ball. }\end{array}$ & - & $\bullet$ & - & $\bullet$ & - & - \\
\hline $\begin{array}{l}\text { Ruppia drepanensis } \\
\text { Tineo }\end{array}$ & - & - & - & - & - & $\bullet$ \\
\hline Ruppia maritima $L$. & - & - & - & - & - & $\bullet$ \\
\hline $\begin{array}{l}\text { Althenia orientalis } \\
\text { (Tzvelev) García } \\
\text { Murillo \& Talavera. }\end{array}$ & • & $\bullet$ & $\bullet$ & $\bullet$ & - & $\bullet$ \\
\hline $\begin{array}{l}\text { Zanichellia obtusifolia } \\
\text { Talavera, García Murillo } \\
\text { \& Smit }\end{array}$ & $\bullet$ & - & - & - & - & $\bullet$ \\
\hline
\end{tabular}


Tabla 3. Especies exóticas y ruderales (citadas por primera vez) halladas en la zonas de estudio. Exotic and ruderal species (first records) found in the study area.

\begin{tabular}{|c|c|}
\hline FAMILIA & TAXONES EXÓTICOS \\
\hline AZOLLACEAE & - Azolla filiculoides Lam. \\
\hline CACTACEAE & - Opuntia megacantha Salm-Dyck \\
\hline AMARANTHACEAE & $\begin{array}{l}\text { - Amaranthus albus } \mathrm{L} . \\
\text { - Amaranthus retroflexus } \mathrm{L} .\end{array}$ \\
\hline MIRTACEAE & - Eucalyptus camaldulensis Dehnh. \\
\hline OXALIDACEAE & - Oxalis pes-caprae $\mathrm{L}$. \\
\hline SOLANACEAE & - Nicotiana glauca R. C. Graham. \\
\hline CUSCUTACEAE & - Cuscuta campestris Yuncker. \\
\hline ASTERACEAE & $\begin{array}{l}\text { - Arctotheca calendula (L.) Levyns. } \\
\text { - Aster squamatus (Sprengel) Hieron. } \\
\text { - Cotula coronopifolia L. } \\
\text { - Xanthium strumarium subsp. cavanillesii (Schouw) D. Löve \& P. Dansereau }\end{array}$ \\
\hline POACEAE & $\begin{array}{l}\text { - Paspalum paspalodes (Michx) Scribner. } \\
\text { - Paspalum vaginatum Swartz. } \\
\text { - Spartina densiflora Brongn. }\end{array}$ \\
\hline \multicolumn{2}{|c|}{ TAXONES RUDERALES CITADOS POR PRIMERA VEZ EN DOÑANA } \\
\hline PAPAVERACEAE & - Papaver roheas $\mathrm{L}$. \\
\hline CARYOPHYLLACEAE & - Spergularia heldreichii Fouc. ex Simon secundus \& P. Monnier. \\
\hline BRASSICACEAE & - Capsella bursa-pastoris (L.) Medicus \\
\hline FABACEAE & $\begin{array}{l}\text { - Medicago murex Willd. } \\
\text { - Melilotus alba Medicus. } \\
\text { - Pisum sativum L. }\end{array}$ \\
\hline EUPHORBIACEAE & $\begin{array}{l}\text { - Euphorbia helioscopia L. } \\
\text { - Euphorbia pterococca Brot. }\end{array}$ \\
\hline GERANIACEAE & - Erodium malacoides (L.) L’Hér. \\
\hline LAMIACEAE & - Marrubium vulgare $\mathrm{L}$. \\
\hline PLANTAGINACEAE & - Plantago serraria $\mathrm{L}$ \\
\hline ASTERACEAE & $\begin{array}{l}\text { - Crepis vesicaria L. subsp. haenseleri (Boiss. ex DC.) P.D. Sell } \\
\text { - Leontodon longirrostris (Finch \& P.D. Sell) Talavera }\end{array}$ \\
\hline LILIACEAE & - Asphodelus fistulosus L. \\
\hline
\end{tabular}

familias observadas según el número de táxones que presentan en el área de estudio.

Respecto a la ordenación de los táxones según sus áreas de distribución, es decir los elementos fitogeográficos, siguiendo a Aparicio et al. (2001) se han encontrado en la zona de estudio táxones: Latemediterráneos (23\%), Amplia distribución (21\%), Paleotemplados (20\%); Mediterráneo-Atlánticos (12\%), Circunmediterráneos $(8 \%)$, Euromediterráneos $(3 \%)$, Ibero-Mauritanos (3\%), Endémicos Peninsulares
(2\%) y Eurosiberianos (2\%), según puede verse en la figura 3. Llama la atención el alto porcentaje de táxones con áreas de distribución grandes y, por el contrario, la escasez de táxones "endémicos".

También, a este respecto, queremos destacar el elevado número de táxones singulares que se han encontrado en las zonas estudiadas y que se muestran en la Tabla 2. En este sentido entendemos por táxones singulares aquellos que están recogidos en las listas rojas, libros rojos 
Tabla 4. Superficie ocupada por las unidades de vegetación reconocidas en la zona de Actuación n 4 (Marisma Gallega). Surface occupied by the vegetation units recognized in Action zone $n^{\circ} 4$ (Marisma Gallega).

\begin{tabular}{|c|c|c|}
\hline \multicolumn{3}{|c|}{ TABLA DE SUPERFICIES (HAS) MARISMA GALLEGA (ZONA DE ACTUACIÓN No 4 ) } \\
\hline UNIDAD & $\begin{array}{l}\text { VUELO 1998-1999 } \\
\text { (superficies en ha) }\end{array}$ & $\begin{array}{l}\text { VUELO 2001-2002 } \\
\text { (superficies en ha) }\end{array}$ \\
\hline Comunidad de Scirpus litoralis y Scirpus maritimus & 307.005 & 320.855 \\
\hline Comunidad de Arthrocnemum macrostachyum con alta cobertura & 488.966 & 480.725 \\
\hline $\begin{array}{l}\text { Comunidad de Scirpus litoralis y Scirpus maritimus con alta } \\
\text { presión ganadera }\end{array}$ & 271.439 & 300.648 \\
\hline $\begin{array}{l}\text { Comunidad de Arthrocnemum macrostachyum con baja cobertura } \\
\text { y alta presión ganadera }\end{array}$ & 1783.701 & 1735.6 \\
\hline Regadíos en pívot & 614.308 & 614.308 \\
\hline Tablas de arroz & 1422.891 & 1422.891 \\
\hline Pinar de Pinus pinea con Pistacia lentiscus y cistáceas diversas & 665.978 & 665.978 \\
\hline Comunidades de Ruppia drepanensis y Callitriche truncata & 8.181 & 5.702 \\
\hline Comunidad de Ranunculus peltatus & 61.476 & 61.725 \\
\hline $\begin{array}{l}\text { Comunidad de Phragmites australis, Typha dominguensis y } \\
\text { Tamarix canariensis }\end{array}$ & 37.897 & 61.611 \\
\hline $\begin{array}{l}\text { Comunidad intermedia con Phragmites australis, Typha } \\
\text { dominguensis y Tamarix canariensis, y Scirpus litoralis y Scirpus } \\
\text { maritimus }\end{array}$ & 122.097 & 112.593 \\
\hline Comunidad dominada por Scirpus maritimus & 52.511 & 31.644 \\
\hline $\begin{array}{l}\text { Restos de antiguos canales con Damasonium alisma y } \\
\text { Ranunculus peltatus }\end{array}$ & - & - \\
\hline
\end{tabular}

o catálogos de especies amenazadas, que tienen un elevado valor desde el punto de vista de la conservación.

De igual forma, se distingue, por contra, el número de especies exóticas que han aparecido en la zona de estudio, recogidas en la Tabla 3 y que representan cerca del $6 \%$ de la flora estudiada. Igualmente, resulta llamativo el elevado numero de especies ruderales que se han encontrado, varias de ellas, las indicadas en la Tabla 3, no habían sido citadas en trabajos previos sobre la flora del Parque Nacional de Doñana.

Finalmente, en relación con la vegetación, las Tablas 4 y 5 muestran la superficie ocupada por cada una de las unidades de vegetación que se reconocieron en los distintos vuelos estudiados. Del mismo modo, las figuras 4 y 5 ilustran la comparación entre las superficies ocupadas por las distintas unidades de vegetación, en los dos momentos considerados.

\section{DISCUSIÓN}

A partir de los datos expuestos sobre la flora del área de estudio, se pueden hacer las siguientes precisiones:

En primer lugar, resaltar la riqueza florística de un lugar, a priori tan poco diverso, como un humedal salino, hecho que se acentúa con el elevado numero de familias encontradas (Fig. 2). Resulta también llamativo el lugar que ocupan las familias Chenopodiaceae ( $4^{\circ}$ lugar) y Characeae $\left(5^{\circ}\right.$ lugar) en la ordenación expuesta. Se trata de familias que, incluso en los catálogos florísticos de humedales, no suelen subir del décimo puesto; hecho que indica la importancia de esta zona desde el punto de vista florístico. En este aspecto incide también el hallazgo de un elevado número de taxones singulares (Tabla 2) en el área de estudio. Todo ello apunta, por tanto en la dirección de que el área de estudio contiene ele- 


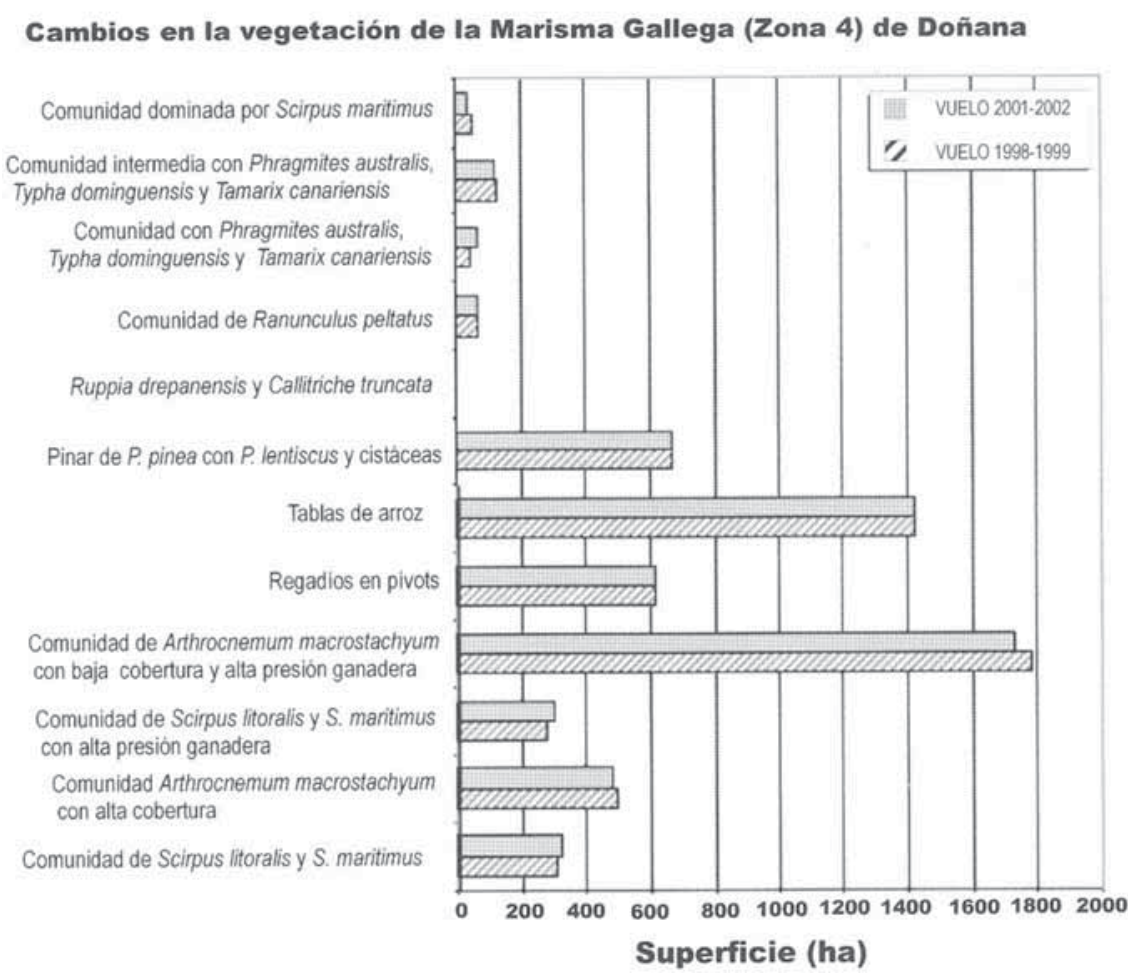

Figura 4. Comparación, entre las superficies ocupadas por las distintas unidades de vegetación consideradas en la zona de actuación $\mathrm{n}^{\circ} 4$ del proyecto de restauración ecológica DOÑNA 2005, en relación con los dos vuelos estudiados (1998-1999/2001-2002). Comparison, between the surfaces occupied by the different vegetation units considered in Action zone $n^{\circ} 4$ of the DOÑANA 2005 Ecological Restoration Project, in relation to both studied flights (1998-1999/2001-2002).

mentos de un gran valor desde el punto de vista de la conservación. Elementos que por si mismos justifican el grado de protección de estas zonas y las medidas de restauración ambiental.

Desde el punto de vista corológico, llama la atención el elevado porcentaje que presentan los elementos florísticos con áreas de distribución extensas (Latemediterráneos, Amplia Distribución y Paleotemplados). Ello se interpreta como la consecuencia de que gran parte de las especies del área de estudio son vegetales acuáticos y el patrón corológico que siguen estos organismos es el de presentar áreas de distribución extensas (Cook, 1983; Santamaría, 2002).

Otro hecho destacable es el elevado número de especies exóticas y ruderales encontradas, la mayor parte de las cuales no aparecían en los catálogos florísticos de Doñana (Cabezudo, 1974; 1975; 1978; Galiano \& Cabezudo, 1976; Castroviejo, 1980; García Murillo, 1993), que fueron realizados a finales del siglo XX y que recogieron de forma exhaustiva las especies vegetales que se encontraban en el Parque Nacional (en la Tabla 3 se incluyen las especies ruderales encontradas y no citadas en ninguna publicación anterior). La interpretación en este caso es que la aparición de tales taxones está indicando un cambio en las condiciones del medio, concretamente un proceso de eutrofización vinculado a las actividades humanas, del que se han hecho eco algunos autores (Fernandez Delgado, 1997; Ferreras Romero et al., 2005). Al incrementarse la concentración de nutrientes, las especies exóticas y ruderales son capaces de competir con ventaja con las especies de los ecosistemas autóctonos, en los que se introducen y desestructuran, limitando las posibilidades de las especies adaptadas a las condiciones originales, que terminan desapareciendo (Jun et al., 2003 y Losová et al., 2006). 
Tabla 5. Superficie ocupada por las unidades de vegetación reconocidas en la zona de Actuación $\mathrm{n}^{0}$ 8. Surface occupied by the vegetation units recognized in Action zone $n^{\circ} 8$.

\begin{tabular}{|c|c|c|}
\hline UNIDAD & $\begin{array}{l}\text { VUELO 1998-1999 } \\
\text { (superficies en ha) }\end{array}$ & $\begin{array}{l}\text { VUELO 2001-2002 } \\
\text { (superficies en ha) }\end{array}$ \\
\hline Salina & 105.221 & 105.221 \\
\hline $\begin{array}{l}\text { Comunidad dominada por Arthrocnemum macrostachyum y } \\
\text { Juncus subulatus }\end{array}$ & 504.282 & 971.092 \\
\hline Fase degradada de sabinares con Pistacia lentiscus & 11.447 & 10.859 \\
\hline Comunidad de Spartina densiflora con Sarcocornia perennis & 13.925 & 16.758 \\
\hline Comunidad de Limoniastrum monopetalum & 2.983 & 3.259 \\
\hline Pastizal halófilo con Tamarix canariensis & 647.222 & 599.085 \\
\hline Comunidad dominada por Juncus subulatus & 113.825 & $\longrightarrow$ \\
\hline $\begin{array}{l}\text { Comunidad de Arthrocnemum macrostachyum sobre salinas } \\
\text { antiguas }\end{array}$ & 106.030 & 106.030 \\
\hline Comunidad de Arthrocnemum macrostachyum con baja cobertura & 1208.374 & 1417.829 \\
\hline $\begin{array}{l}\text { Comunidad de Ruppia drepanensis, Chara spp. y Arthrocnemum } \\
\text { macrostachyum }\end{array}$ & 235.234 & 414.944 \\
\hline Comunidad dominada por Juncus subulatus y Scirpus littoralis & 309.121 & - \\
\hline Comunidad de Spartina densiflora densa & 108.234 & 106.872 \\
\hline $\begin{array}{l}\text { Comunidad Sarcocornia perenne, Halimione portulacoides e } \\
\text { Inula crithmoides }\end{array}$ & 33.101 & 33.101 \\
\hline $\begin{array}{l}\text { Comunidad de Arthrocnemum macrostachyum con influencia } \\
\text { mareal }\end{array}$ & 188.434 & 106.814 \\
\hline Comunidad de Arthrocnemum macrostachyum con alta cobertura & 626.537 & 341.046 \\
\hline $\begin{array}{l}\text { Lucio artificial con comunidades de Ruppia drepanensis y Chara } \\
\text { spp. }\end{array}$ & 35.390 & 32.224 \\
\hline Veta conchífera con Eucalyptus camaldulensis & 0.457 & 0.457 \\
\hline Veta conchífera con Opuntia ficus-indica & 0.538 & 0.538 \\
\hline Tablas de salinas (comunidades de Althenia orientalis) & 37.775 & 37.775 \\
\hline Comunidad de Scirpus litoralis y Scirpus maritimus & 49.845 & 52.129 \\
\hline
\end{tabular}

Cambios en la vegetación de la marisma salada (Zona 8) de Doñana

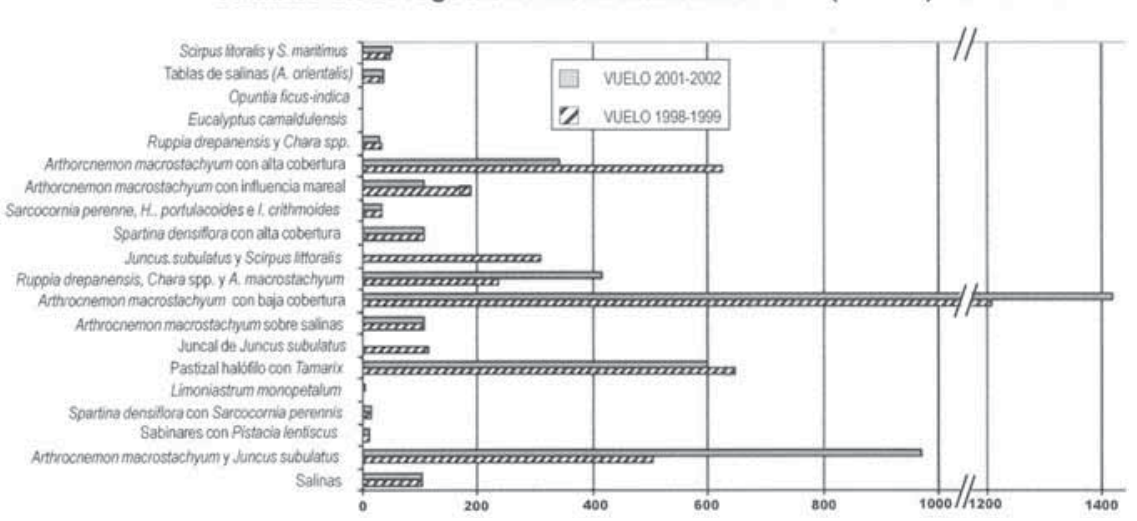

Figura 5. Comparación, entre las superficies ocupadas por las distintas unidades de vegetación consideradas en la zona de actuación $\mathrm{n}^{\circ} 8$ del proyecto de restauración ecológica DOÑANA 2005, en relación con los dos vuelos estudiados (1998-1999/2001-2002). Comparison, between the surfaces occupied by the different vegetation units considered in Action zone $n^{o} 8$ of the DOÑANA 2005 Ecological Restoration Project, in relation to both studied flights (1998-1999/2001-2002). 
Respecto a la información expuesta sobre la vegetación. Las dos zonas estudiadas presentan una cubierta vegetal con una heterogeneidad bastante acusada. Sin embargo, tras comparar los datos de las Tablas 4 y 5, y examinar las figuras 4 y 5; aunque se aprecian diferencias entre la superficie ocupada por algunas unidades en los dos vuelos, estas son de una magnitud menor y las achacamos a la variabilidad interanual de la vegetación de la marisma, ya señalada por diversos autores (Cobo et al., 2002; Marín \& García Novo, 2005), mas que al efecto de las obras de restauración. Por tanto, para apreciar el efecto de los trabajos de restauración, habrá que considerar vuelos más recientes.

Finalmente señalar que, a la vista de lo expuesto, tanto el empleo de un catálogo florístico, como el conocimiento de la ubicación y superficie ocupada por las distintas comunidades vegetales, considerados desde un punto de vista diacrónico, pueden resultar unos instrumentos excelentes para evaluar el éxito de este tipo de trabajos de restauración ambiental.

\section{AGRADECIMIENTOS}

Los trabajos referidos en este artículo se han desarrollado dentro del proyecto DOÑANA 2005 del Ministerio de Medio Ambiente. Asimismo, los autores desean agradecer al personal del Parque Nacional de Doñana y de la Estación Biológica de Doñana (CSIC) las facilidades dadas para la realización de nuestras investigaciones.

\section{REFERENCIAS}

APARICIO, A., C. PÉREZ PORRAS y G. CEBALLOS. 2001. Inventario y caracterización florística de los 'Bosques-isla' de la campiña de Cádiz. Consejería de Medio Ambiente, Junta de Andalucía. Sevilla. 207 pp.

BAÑARES, A., G. BLANCA, J. GÜEMES, J. C. MORENO SANZ y S. ORTIZ. 2003. Atlas y libro rojo de la flora vascular amenazada de España. Ministerio de Medio Ambiente. Madrid. 1067 pp.

BAYÁn, B. y E. ALONSO. 2006. Proyecto de restauración hidroecológica Doñana 2005. Datos básicos 2006. Doñana 2005, 7:1-15.

BERNUÉS, M. 1990. Limnología de los sistemas acuáticos superficiales del Parque Nacional de Doñana. Tésis Doctoral. Universidad Autónoma de Madrid. 242 pp.

BLANCA, G., B. CABEZUDO, J. E. HERNÁNDEZ-BERMEJO, C. M. HERRERA, J. MUÑOZ y B. VALDÉS (eds.) 1999-2000. Libro rojo de la Flora silvestre amenazada de Andalucia. Tomo I: Especies en peligro de extinción. Tomo II: Especies vulnerables. Consejería de Medio Ambiente. Sevilla. 302 pp. y 375 pp.

BRAUN-BLANQUET, J. 1979. Fitosociología. Bases para el estudio de las comunidades vegetales. Ediciones Blume, Madrid. 820 pp.

CABEZUDO, B. 1978. Plantas de la Reserva Biológica de Doñana II. Lagascalia, 8(2): 166-182.

CABEZUDO, B., S. TALAVERA, G. BLANCA, C. SALAZAR, M. CUETO, B. VALDÉS, J. E. HERNÁNDEZ BERMEJO, C. M. HERRERA, C. RODRÍGUEZ y D. NAVAS. 2005. Lista roja de la Flora Vascular de Andalucía. Consejería de Medio Ambiente. Junta de Andalucía. Sevilla. 126 pp.

CASTROVIEJO, S., VALDÉS-BERMEJO, E., RIVAS-MARTÍNEZ, S. y COSTA, M. 1980. Novedades florísticas de Doñana. Anales Jardín Botánico de Madrid 36: 203-244.

CIRUJANO, S., C. MONTES, P. MARTINO, S. ENRIQUEZ y P. GARCÍA MURILLO. 1988. Contribución al estudio del género Riella Mont. (Sphaerocarpales, Riellaceae) en España. Limnetica, 4: 41-50.

CIRUJANO, S. M., VELAYOS, F. CASTILLA y M. GIL. 1992. Criterios botánicos para la valoración de las lagunas y humedales españoles (Península Ibérica e Islas Baleares). ICONA. Madrid. 456 pp.

COBO, M. D., E. SÁNCHEZ GULLÓN y P. GARCÍA MURILLO. 2002. Flora y Vegetación. In: Parque Nacional de Doñana García Canseco (ed.): 108-174. Canseco Editores SL. Talavera de la Reina.

COMELLES, M. 1985. Clave de identificación de las especies de carófitos de la Península Ibérica. Asociación Española de Limnología. Barcelona. 35 pp.

COOK, C. D. K. 1983. Aquatic plants endemic to Europe and the Mediterranean. Botanische Jahrbücher Syst., 103: 539-582

DUARTE, C., C. MONTES, S. AGUSTI, P. MARTINO, M. BERNUES, y J. KALFF. 1990. Biomasa de macrofitos acuaticos en la marisma del Parque Nacional de Doñana (SO. España): importancia y 
factores ambientales que controlan su distribucion. Limnetica, 6: 1-12.

ESPINAR, J. L. 2000. Distribución espacial y temporal de las comunidades de macrófitos acuáticos de la Marisma Salada del Parque Nacional de Doñana. Tesis de Licenciatura. Universidad de Sevilla. 126 pp.

ESPINAR J. L., L. V. GARCÍA, P. GARCÍA MURILLO \& J. TOJA. 2002. Submerged macrophyte zonation in a Mediterranean salt marsh: a facilitation effect from estabilished helophytes?. Journal of Vegetation Science, 13: 1- 15.

FERNÁNDEZ-DELGADO, C. 1997. Conservation management of a European natural area: Doñana National Park, Spain. In: Principles of conservation biology. 2nd edition. Meffe G. K. \& C. R. Carroll (eds.): 458-467. Sinauer Associates, Sunderland, Massachusetts.

FERRERAS ROMERO, M., J. FRÜND y J. MÁRQUEZ RODRÍGUEZ. 2005. Sobre la situación actual de Lestes macrostigma (Eversmann, 1836) (Insecta: Odonata) en el área de Doñana (Andalucía, sur de España). Bol. Asoc. Esp. Ent., 29: 41-50.

GALIANO, E. F. y B. CABEZUDO. 1976. Plantas de la Reserva Biológica de Doñana (Huelva). Lasgascalia, 6: 117-176.

GARCÍA MURILLO, P., M. BERNÚES, y C. MONTES, 1993. Los macrofitos acuáticos del Parque Nacional de Doñana (SW España). Aspectos florísticos. Actas VI Congreso Español de Limnología. Granada: 261-267.

GARCÍA NOVO, F. 1997. The ecosystems of Doñana Nacional Park. In: The ecology and conservation of European dunes. García Novo, F., R.M.N. Carwford \& M.C. Díaz Barradas (eds.): 77-116. Universidad de Sevilla. Sevilla.
HERNÁNDEZ BERMEJO J., A. PUJADAS SALVÁ y M. CLEMENTE MUÑOZ. 1994. Catálogo general de las especies de recomendada protección para Andalucía (endémicas, raras y amenazadas de Extinción). In: Protección de la Flora en Andalucía. J. Hernández Bermejo y M. Clemente Muñoz (eds.) 43-67. Junta de Andalucía. Consejería de Cultura y Medio Ambiente. Agencia de Medio Ambiente. Sevilla.

KÜHN, I., R. BRANDL, R. MAY \& ST. SLOTZ. 2003. Plant distribution patterns in Germany-Will aliens match natives? Feddes. Repertorium, 114: 559-573.

LOSOVÁ, Z., M. CHYTRY, I. KÜHN, O. HÁJECK, V. HORÁKOVÁ, P. PYSEK \& L. TICHÝ. 2006. Patterns of plant traits in annual vegetation of made-man habitats in central Europe. Perspectives in Plant Ecology, Evolution and Systematics, 8: 69-81.

MARÍN, C. y F. GARCÍA NOVO. 2005. Doñana Agua y Biosfera. Confederación Hidrográfica del Guadalquivir. Ministerio de Medio Ambiente. Sevilla. 366 pp.

NARBONA, C. 2006. Vuelta a la naturalidad de Doñana. Quercus Edición especial Doñana 2005Corredor Verde del Guadiamar: 1.

RIVAS-MARTÍNEZ, S., M. COSTA, S. CASTROVIEJO y E. VALDÉS. 1980. La vegetación de Doñana (Huelva, España). Lazaroa, 2: 5-189.

SANTAMARÍA. L. 2002. Why are most aquatic plants widely distributed? Dispersal, clonal growth and small scale heterogeneity in a stressful environment. Acta Oecologica, 23: 137-154.

VALDÉS, B., S. TALAVERA y E. F. GALIANO (Eds.). 1987. Flora vascular de Andalucía Occidental. Ketres. Barcelona. 3 vols. 485 pp., 640 pp. y $555 \mathrm{pp}$. 